\title{
WiQoSM: An Integrated QoS-Aware Mobility and User Behavior Model for Wireless Data Networks*
}

\author{
Giovanni Resta $^{\dagger} \quad$ Paolo Santi ${ }^{\dagger}$
}

\begin{abstract}
Modeling mobility and user behavior is of fundamental importance in testing the performance of protocols for wireless data networks. While several models have been proposed in the literature, none of them can at the same time capture important features such as geographical mobility, user generated traffic, and wireless technology at hand. When collectively considered, these three aspects determine the user-perceived QoS-level, which, in turn, might have an influence on mobility of those users (we call them QoSdriven users) who do not display constrained mobility patterns, but they can decide to move to less congested areas of the network in case their perceived QoS-level becomes unacceptable.

In this paper, we introduce the WiQoSM model which collectively considers all the above mentioned aspects of wireless data networks. WiQoSM is composed of $i$ ) a user mobility model, ii) a user traffic model, iii) a wireless technology model, and iv) a QoS model. Components $i$ ), ii), and $i i i$ ) provide input to the QoS model, which, in turn, can influence the mobility behavior of QoS-driven users. WiQoSM is very simple to use and configure, and can be used to generate user and traffic traces at the APs composing a wireless data network.
\end{abstract}

WiQoSM is shown to be able to generate traces which resemble statistical features observed in traces extracted from real-world WLAN deployments. Furthermore, WiQoSM

*A preliminary version of this paper appeared in Proc. ACM/IEEE International Symposium on Modeling, Analysis and Simulation of Wireless and Mobile Systems (MSWiM), 2006.

${ }^{\dagger}$ Istituto di Informatica e Telematica del CNR, Pisa, ITALY. email:\{giovanni.resta, paolo.santi\}@it.cnr.it 
has the nice feature of allowing fine tuning of disjoint set of parameters, in order to influence different statistical properties of the generated traces, and of providing the network designer with a high degree of flexibility in choosing network parameters such as number of users and APs, wireless channel technology, traffic mix, and so on. Given the above features, WiQoSM can be a valuable tool in the simulation of wireless data network protocols.

Index terms: Mobility modeling, user behavior modeling, QoS-driven mobility, wireless data networks.

\section{Introduction}

Thanks to the increasing popularity of 802.11-based products, wireless data networks have become widespread in recent years: medium- to large-scale WLAN deployments are nowadays common in campuses, enterprises, and so on, and also public areas such as airports, shopping malls, and parks, are becoming increasingly covered by wireless access points (APs). The proliferation of wireless data networks is expected to increase even further when the recently introduced wireless Mesh technology will become mature [7].

In order to fulfill the promise of ubiquitous connectivity, forthcoming wireless data networks must face several challenges, including the design of appropriate congestion control mechanisms and interference mitigation protocols, whose performance should be carefully optimized depending on the scenario at hand. Given the high costs of on-the-field testing, simulation is often the preferred option to test the performance of network protocols.

There are several factors that influence performance of a wireless data network, including infrastructure (number and location of the APs), number of active users, their mobility and traffic patterns, the wireless technology at hand, and so on. This implies that, in order to obtain an accurate estimation of the performance of a certain protocol for wireless data networks, none of these aspects should in principle be neglected at the simulation stage. Furthermore, a mobility and user behavior model should be relatively simple, while at the same time general enough to allow the simulation of a wide range of network settings.

Despite the considerable amount of research devoted to mobility and user behavior modeling 
in recent years, to the best of our knowledge none of the models introduced in the literature so far considers all the above mentioned aspects at the same time. On one hand, we have synthetic models aimed at resembling user registration patterns observed in a certain realworld deployment (e.g., the Dartmouth college [13, 17, 20, 21], or the ETH Zurich campus [27]). While these models allow some flexibility in the choice of some parameters, such as number of users and APs, they do not consider user traffic patterns. Most importantly, these models are tailored to a very specific network deployment, and cannot be used to test the performance of wireless data network protocols in different scenarios. On the other hand, we have 'general purpose' random models that typically account for only one of the above mentioned aspects influencing wireless data network protocols performance. For instance, we have separate synthetic models for user mobility (such as the well-known random waypoint model [15]), traffic pattern, radio channel characteristics, and so on. However, these models (especially for what concerns mobility) are often considered too general, and not representative of any real-world scenario.

In light of the above, the need of more realistic, yet simple, mobility and user behavior models for testing the performance of wireless data network protocols, which is mentioned in [3] as one of the challenges related to the realization of ubiquitous wireless connectivity, has not been satisfied yet. In this paper, we make a first step in this direction introducing WiQoSM (Wireless QoS-aware Mobility model), an integrated mobility, user behavior, and wireless technology model which can be used in the performance evaluation of wireless data network protocols.

A salient and novel feature of WiQoSM is that it allows mimicking a situation in which a fraction of users displays constrained movement patterns (e.g., because they have to move to a certain specific location within a certain time), while the remaining users are mainly stationary, but they could decide to move to another location in case their perceived QoS degrades considerably. In the remainder of this paper, we call users of the former type mobile users, and users of the latter type QoS-driven users. We believe distinguishing users into mobile and QoS-driven is representative of many WLAN or wireless Mesh deployments in public areas such as airports, parks, shopping malls, and so on, as acknowledged also in [2, 25]. For instance, consider the case of a public park. In this situation, a fraction of users might be 
seated somewhere in the park, using their wireless devices to browse the Web, sending emails, downloading music files to listen with the iPod, and so on. For this class of users, what is relevant is the perceived QoS: as long as the QoS is satisfactory, there is no reason to move to another location. However, if the QoS degrades below an acceptable level, a typical user of this class may decide to look for a better location in the park, becoming stationary again when the perceived QoS is restored to an adequate level. It is not difficult to imagine similar scenarios in other environments such as airports, shopping malls, campuses, and so on. Finally, we also include in the model a (small) fraction of stationary users, whose location is fixed even in presence of low QoS levels. Stationary users account for the small fraction of users with prevalence 1 (i.e., users who are always registered with the same AP when active) which has been observed in real-world traces extracted from enterprise [4] and campus [27] environments.

WiQoSM generates user and traffic traces which can be used in combination with custom simulators to evaluate the performance of a specific wireless data network protocol. We believe using WiQoSM-generated traces can considerably improve the accuracy of wireless data network protocols' performance estimation, in contrast to the current habit of using purely random approaches (e.g., the traffic observed at an AP is generated uniformly at random between an upper and lower bound).

Another interesting feature of WiQoSM is that the generated traces are accompanied by a set of metrics such as balance index, user prevalence and persistence in AP association, distribution of session duration, and so on, that can be used to assess relevant features of the generated traces. Trace statistics are very useful when trying to tailoring WiQoSM to a specific application scenario (e.g., campus networks), for which at least some of these statistics are known. This is what we have done in Section 5, where we describe how to tune WiQoSM parameters in order to obtain traces which resemble those collected in an enterprise [4] and campus [27] environment.

The rest of this paper is organized as follows. In Section 2, we survey related work and discuss the main contributions of this paper. In Section 3, we introduce the WiQoSM mobility and user behavior model, and in Section 4 we describe how WiQoSM can be used to generate user/traffic traces for wireless data networks. In Section 5 we assess WiQoSM ability of producing traces whose statistical features resemble those observed in traces extracted from some 
real-world WLAN deployments. In Section 6, we evaluate through WiQoSM the effect on trace statistics of having a different fraction of QoS-driven users in the network. Finally, Section 7 concludes and discusses possible ways of extending/improving our model.

\section{Related work and contribution}

The characterization of user behavior in wireless data networks has been subject of intensive research in recent years. By analyzing user traces collected at the various access points in campuses [16, 18], corporate buildings [4], or conferences [1], typical user behaviors have been analyzed, and phenomena such as different popularity levels of the APs, occasional network congestion, low correlation between the number of users and load, and so on, have been observed. More recently, some authors have derived synthetic models of user behavior which try to mimic the observed user behavior in a certain environment. This is the case, for instance, of the ModelT model proposed in [13], which is based on the traces collected from the Dartmouth College campus over a period of 2 years. This model has been recently extended to account for spatio-temporal correlation in the user registration patterns [21]. Other examples of synthetic mobility models based on real-world traces are proposed in [17, 20, 27]. While allowing the network designer to have a certain degree of freedom in setting some network parameters such as number of users and access points, synthetic models based on real-world traces have the disadvantage of being representative of a very specific scenario (the one from which the traces were collected). Furthermore, these models are built to capture the characteristics of existing or past wireless networks, and it is not clear to what extent these models are representative of future wireless network deployments.

Another related active area of research in recent years is the analysis of existing 'general purpose' synthetic mobility models, such as the random waypoint (RWP) and the random direction model, which, mostly due to their simplicity, are the most commonly used in the simulation of wireless ad hoc network protocols. Recent studies have characterized the long term node behavior in these types of mobile networks [5, 19, 22, 24, 28, 29], outlining some undesired phenomena such as node concentration in the center of the deployment region [5], and long term decay of the average nodal speed [28]. These phenomena could impair the 
accuracy of simulations based on these synthetic mobility models, essentially because the initial network conditions can be very different from the long term network conditions. Another acknowledged weakness of random waypoint/direction -like mobility is that it is too general, and not representative of any real-world scenario. In order to address some of the criticisms raised against random waypoint/direction mobility, some authors have recently proposed more realistic and/or more accurate synthetic mobility models, such as the obstacle model proposed in [14], and those proposed in [6, 12, 23, 29]. For a survey on mobility models for wireless ad hoc networks the reader is referred to [8].

Despite the considerable amount of research efforts referred above, to the best of our knowledge none of the existing synthetic models of user behavior in wireless networks jointly considers the three aspects that influence performance of wireless data network protocols, i.e., $i$ ) geographical user mobility, $i i)$ user traffic patterns, and iii) wireless technology at hand. Furthermore, none of the existing models accounts for the fundamental observation that a fraction of users might not display constrained movement patterns, but they could decide to move to a better location in case their perceived QoS level drops below an acceptable level. We want to remark that the fact that some users might be willing to move to another location to improve their QoS has already been observed in [25], and explicitly suggested as a method to alleviate congestion in [2]. In the latter paper, the authors introduce the concept of network-directed roaming, which can be briefly explained as follows: if the required QoS cannot be guaranteed to a certain user, the network itself indicates to the user where to roam in order to obtain the desired level of service. This technique, combined with explicit channel switching, is shown to considerably improve load balancing in the network.

The main contribution of this paper is the proposal of an integrated QoS-aware mobility and user behavior model that jointly considers $i$ ), ii), and iii) above. Our proposed model, WiQoSM, is composed of four components: a mobility model, a user traffic model, a wireless technology model, and a QoS model. The mobility model implements weighted random waypoint mobility, where the next waypoint is chosen according to an AP popularity metric. The fact that different APs in a wireless data network display different popularity degrees (i.e., number of registered users) has been recently observed in different types of wireless data networks $[4,18,26]$. As such, we believe accounting for the popularity degree when choosing the next 
waypoint contributes to improving the accuracy of our model. To model user traffic behavior, we classify users into three classes depending on the amount of load offered to the network. Note that this classification of users based on the offered load is (in principle) orthogonal to their classification into mobile/QoS-driven/stationary. The third fundamental building block of WiQoSM is the wireless technology model, which is in turn composed of a channel access and a radio propagation model. Currently, we have implemented two channel access methods (data polling and time polling), and two radio propagation models (log-distance path loss and log-normal shadowing), but other models can be easily integrated in our framework. The mobility, user traffic, and wireless technology models provide input to the QoS model, which estimates the degree of satisfaction for each user in the network according to a simple criteria. If a user is QoS-driven, he/she can decide to change its AP association and/or physical location depending on the perceived QoS-level. The block diagram of our WiQoSM model is reported in Figure 1.

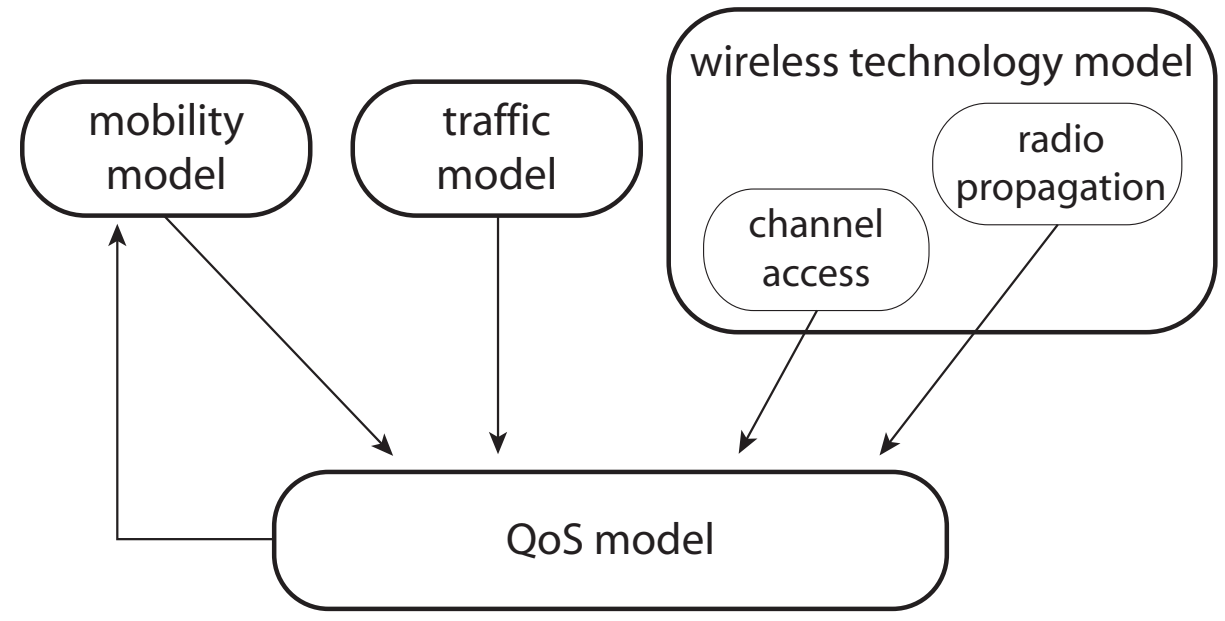

Figure 1: Block diagram of the WiQoSM model.

Observe that the need of estimating the degree of user satisfaction complicates a lot the model definition, since aspects such as user offered load and channel access method have to be carefully modeled. To keep the complexity of our proposed model to a reasonable level, we have decided to simplify the mobility part as much as possible, while at the same time accounting 
for relevant aspects such as degree of AP popularity. This explains our choice of modeling user movements according to the well-known RWP model, suitably modified to choose the waypoints according to an AP popularity metric.

\section{WiQoSM}

In this Section we introduce our mobility and user behavior model, which we call WiQoSM (Wireless QoS-aware Mobility). The model is composed of four main components: mobility modeling, user traffic modeling, wireless technology modeling, and QoS modeling (recall Figure 1). We describe each of these components in a separate subsection.

\subsection{Mobility model}

As mentioned in the previous sections, in WiQoSM we assume that a certain fraction $f$, with $0 \leq f \leq 1$, of the $n$ network users is QoS-driven (QoS-driven users), another (small) fraction $s$, with $0 \leq s \leq 1$, is stationary, while the remaining $(1-f-s) \cdot n$ users display constrained movement patterns (mobile users).

We want to remark that what is relevant in the above categorization of non-stationary users is whether their movement pattern is constrained or not. In other words, mobile users might be sensitive to QoS level as QoS-driven users, but their distinguishing feature is that they cannot modify their movement behavior in case the perceived QoS level is low. An example of this type of user might be a person (call her Alice) using a VoIP application while walking to the office. While definitely sensible to QoS degradation, Alice's movement is heavily constrained, and cannot be changed in response to QoS degradation (if we exclude short detours from the main trajectory to the office which, however, are likely to have little effects on QoS). Note that Alice might undertake other types of countermeasures if the QoS level degrades too much, such as dropping the call and re-calling at a later time ${ }^{1}$.

Movement of QoS-driven users obeys the following rules. Users in this class are mostly stationary, i.e. they are not willing to move unless their perceived QoS level drops below an

\footnotetext{
${ }^{1}$ This feature is currently not implemented in WiQoSM.
} 
acceptable threshold. The determination of whether a certain QoS level is acceptable for the user is left to the QoS model (see Section 3.4).

Since we assume that QoS-driven users are 'lazy', and that the only reason forcing them to move is low QoS level, in our model we assume that an unsatisfied QoS-driven user first tries to do a 'virtual movement' by simply changing AP association. Using the terminology introduced in [2], we call this action channel switching.

In case channel switching is possible, user $u$ changes AP association, selecting the AP which provides him/her the best QoS. Estimating the QoS level achieved by surrounding APs is not immediate, since the user perceived QoS depends not only on the quality of the wireless channel connecting to the AP, but also on the load offered by the other users registered at that AP. Hence, similarly to what proposed in [2], the policy for selecting the new AP should be based on a metric that accounts for both signal strength and observed load at the AP. To reduce the complexity of the simulator, in WiQoSM we have opted for using a simpler metric for selecting the new AP, which is based on the observed load at the AP in the last log interval (default setting of the log interval is $60 s$ ). More specifically, user $u$ selects as the AP with which trying a new association the one with the lowest observed load.

In case channel switching is not possible (because there is no overlapping of APs in the current user's position, or because also the other AP is overloaded and QoS level remain low), an unsatisfied QoS-driven user decides to do a physical move. Again, there are many different options to model movement of an unsatisfied user. To keep the model simple, we assume that unsatisfied QoS-driven users move according to the same rules governing the movement of mobile users, i.e. AP popularity weighted RWP movement (see below). However, the popularity rule is reversed, i.e. QoS-driven users tend to move (according the a probability distribution which is obtained by inverting the one used for governing mobile user movements) to less popular locations. Since estimating AP popularity might be difficult for a user, we also implement a different rule for movement of QoS-driven users, i.e. choosing a new destination AP (waypoint) uniformly at random. We call the former rule unpopular movement, and the second rule oblivious movement (which, we recall, apply only to QoS-driven users).

Let us now consider mobile users. In order to reduce the complexity of our model, we assume that mobile users follow a weighted waypoint mobility model similar to that proposed 
in $[12]^{2}$. Mobile users alternate between pause times at waypoints and traveling periods between successive waypoints. Initially, a user is assigned a certain waypoint according to a weighted, non uniform distribution which resembles the different popularity of APs. The fact that APs in a wireless data network display different popularity degrees is well documented in the literature $[1,4,18]$. In our model, we initially assign each AP $a$ with a popularity degree $p(a) \in\left[p_{\min }, p_{\max }\right]$ according to a probability distribution resembling a bounded power law (as suggested in [13]). Alternatively, it is possible to manually specify popularity degrees for each AP, or to use different probability distributions for AP popularity.

When selecting the initial or a new waypoint for a certain mobile user, first an AP is selected with a probability proportional to its popularity, then a waypoint is selected uniformly at random within the coverage area of the selected AP. The pause time at waypoints is chosen according to a Poisson distribution with intensity $\lambda_{p}$. In order to avoid degenerate situations of excessively long or short pause times, we have imposed an upper and lower bound to the pause time at the waypoints. Note that other pause time distributions can be chosen and easily included in our mobility model, such as the log-normal distribution observed in [17] for VoIP users. Differently from the model used in [17], in which intermediate waypoints between the origin and the destination of a movement are randomly chosen (resulting in a non-linear trajectory), in WiQoSM the trajectory between consecutive waypoints is a straight line. Velocity is chosen uniformly at random in an interval $\left[v_{\min }, v_{\max }\right]$.

Initial location of QoS-driven and stationary users are also chosen according to the AP popularity-based method described above.

\subsection{User traffic model}

In WiQoSM, users are divided into three different classes of offered load: low, medium, and high load. The lowest class of traffic accounts for users who are using the network for emailing,

\footnotetext{
${ }^{2}$ We are aware that more realistic mobility models such as, for instance, the obstacle model of [14], could have been used in WiQoSM. However, this would have increased a lot the complexity of the simulator and the simulation running time. For this reason, we have opted for a simple model such as random waypoint mobility, but enriched with 'more realistic' salient features such as weighted choice of waypoints according to AP popularity.
} 
chatting, and light web browsing. The average bandwidth requirement of this class of users is a tunable parameter, but the actual bandwidth requirement is very variable, since users in this class typically generate bursty traffic. In the example of WiQoSM utilization reported in Section 5, the average bandwidth requirement of this class of users is set to $64 \mathrm{~Kb} / \mathrm{sec}$.

The medium class of traffic accounts for users who are using the network for intensive web browsing, file downloading, audio streaming, and so on. The average user bandwidth requirement in this case is also a tunable parameter, and the generated traffic displays a more uniform pattern. To maintain the complexity of the simulator at a reasonable level, we assume users in this class generate CBR (Constant Bit Rate) traffic. In the study reported in the following, we have set the average bandwidth requirement of medium users to $256 \mathrm{~Kb} / \mathrm{sec}$.

Finally, the highest class of traffic accounts for users who make an intensive use of the network, such as video streaming. The average user bandwidth requirement is again a tunable parameter. Similarly to the case of medium traffic, also high traffic users typically display a relatively uniform bandwidth usage. Hence, we assume CBR traffic for these users, with a rate which is set to $2 \mathrm{Mb} / \mathrm{sec}$ in the study reported in the following.

For the three classes of traffic, actual load generated by a user is selected either uniformly at random within lower and upper bounds centered at the average bandwidth requirement, or according to a Gaussian distribution with a certain std centered at the average bandwidth requirement. For simplicity, in the simulation study reported in sections 5 and 6 all the users generate traffic at a rate corresponding to the average bandwidth requirement of their class.

The allocation of users to the various traffic classes is performed according to a certain mix $l, m, h$, where $l$ is the fraction of low traffic users, $m$ is the fraction of medium traffic users, and $h=1-(l+m)$ is the fraction of heavy traffic users. These fractions can be different for QoS-driven, mobile, and stationary users.

\subsection{Wireless technology model}

The wireless technology model is composed of two sub-models: radio channel model and channel access model.

The radio channel model is as follows. For given user $u$ and $\operatorname{AP} a$, we estimate the quality 
of signal between $u$ and $a$ according to a radio propagation model. In the current version of the model, we have considered log-distance path loss and log-normal shadowing propagation, but other propagation models can easily be integrated into WiQoSM. The quality of signal is used to determine the achievable data rate between $u$ and $a$, if $u$ is actually within the coverage range of AP $a$. We have considered the eight possible data rates available in 802.11a, i.e. 6, 9, $12,18,24,36,48$ and $54 \mathrm{Mbs}$. The nominal radio range of an AP at the various data rates is set according to what reported in the data sheets of the CISCO Aironet 1240AG access point [9]. The nominal ranges are used in combination with the log-distance path loss radio propagation model to determine the highest possible available data rate between $u$ and $a$. In case of lognormal shadowing, the distance between $u$ and $a$ cannot be directly converted into a data rate value, due to the random component in the signal attenuation caused by shadowing. We recall that in the log-normal shadowing model, the attenuation of the received signal (in $d B$ ) at a certain distance $d$ from the transmitter is modeled as the sum of a deterministic quantity which obeys log-distance path loss with a certain path loss exponent $\alpha$, and of a random component which is modeled as a random variable with Normal distribution, zero mean, and variance $\sigma$. We can interpret this model as converting the actual distance between transmitter and receiver into a 'virtual distance', which is computed as the distance between the sender and the receiver if attenuation of the signal (computed according to lognormal shadowing model) was governed only by log-distance path loss. Note that the 'virtual distance' can be either smaller than (when the random component of the attenuation is positive) or larger than (when the random component of the attenuation is negative) the actual distance. Hence, in case of log-normal shadowing model the geographical distance between $u$ and $a$ is converted into 'virtual distance' accounting for the actual signal attenuation between $u$ and $a$, and the highest available data rate is obtained by comparing this 'virtual distance' with the nominal radio ranges.

As mentioned in the Introduction, WiQoSM implements two channel access methods: data polling and time polling. Data polling is similar to the $802.11 \mathrm{a} / \mathrm{b} / \mathrm{g}$ MAC layer with PCF coordination: registered users are polled by the AP. In case a polled user $u$ has a packet to send/receive to/from the AP $a$, the packet is sent at the highest possible data rate available between $u$ and $a$. If a polled user has nothing to send/receive, the next registered user is polled, and so on. Note that, from a high level point of view, there is little difference between DCF 


\begin{tabular}{|c|c|}
\hline Nominal (Mbs) & Actual (Mbs) \\
\hline \hline 54 & 25 \\
\hline 48 & 24 \\
\hline 36 & 19.5 \\
\hline 24 & 16 \\
\hline 18 & 12.8 \\
\hline 12 & 9.6 \\
\hline 9 & 7.2 \\
\hline 6 & 5,4 \\
\hline
\end{tabular}

Table 1: Actual data rates achieved with 802.11a.

and PCF coordination in 802.11, since DCF is designed to give the same long term probability of accessing the channel to contenders [11]. Hence, our data polling model is representative also of $802.11 \mathrm{a} / \mathrm{b} / \mathrm{g}$ networks with DCF coordination.

In order to keep the complexity of simulation at a reasonable level, in WiQoSM we have not implemented any exchange of control messages. However, we have accounted for the overhead caused by control message exchange and channel access time by using an actual data rate for sending packets which is significantly lower than the nominal data rate. In particular, we have used the actual data rates reported in Table 1, which are obtained from the measurements reported in $[10]$.

The current implementation of the $802.11 \mathrm{a} / \mathrm{b} / \mathrm{g}$ MAC layer is known to have major inefficiencies. In particular, both DCF and PCF access methods suffer when registered users have different data rates, because the slowest user reduces the throughput of the other users down to the value of the slowest user ${ }^{3}$. This performance anomaly, which has been observed in [11], is caused by the data polling approach, in which a user, once gained access to the channel, transmits a packet, independently of his/her data rate. Hence, the time allotted to each user can be very different, and slower users tend to use the channel for longer times, driving down the throughput of faster users.

The second access model used in WiQoSM, time polling, solves this problem by assigning to each polled user a time slot, instead of whatever time is necessary to send a packet. With this approach, all the users gain access to the channel for an approximately equal share of time, and faster users can transmit more packets than slower ones. Time polling resembles the basic functioning of the 802.11e MAC extension for QoS support, which is in the final stage of

\footnotetext{
${ }^{3}$ This is true under the assumption that all the registered users always have a packet to send in their queues.
} 
standardization.

A final consideration about channel access in WiQoSM is that we assume adjacent APs use different (orthogonal) channels, so that interference between users registered at adjacent APs is not an issue.

\subsection{QoS model}

Modeling QoS is the most involved component of our model. Note that, in practice, user perceived QoS-level depends on a number of factors, such as user application, network-level policies, number of active users and their traffic patterns, and so on. As a consequence of that, an accurate estimate of user-perceived QoS level can be done only by tightly integrating the QoS component of WiQoSM into the specific simulator used to test the performance of the network protocol at hand. An alternative, which is pursued here, is to provide an approximate estimate of user-perceived QoS level in a stand-alone fashion, through the use of a simple and quite general QoS model.

To determine whether the QoS level is acceptable for a certain user $u$, we measure the number of buffer overflow/underflow (depending on whether we are sending or receiving packets) experienced by $u$ during the last $\Gamma$ seconds, where $\Gamma$ is a tunable parameter which can be used to smooth temporary variations in the QoS level. The status of the buffer is checked every $\gamma$ seconds, with $\gamma \ll \Gamma$, where a check is successful if there is no overflow/underflow, unsuccessful otherwise. If at least a fraction of $r q(u)$ checks are successful, where $r q(u)$ is a tunable parameter modelling the minimum QoS level that user $u$ considers acceptable, then $u$ is satisfied, otherwise it is unsatisfied.

We are aware that the number of buffer overflow/underflow gives only a partial view of what is the user perceived QoS, since other metrics such as delay, jitter, end-to-end throughput, and so on, are typically used to measure QoS. However, we believe that the above metric gives a sufficiently accurate estimate of QoS, without requiring explicit knowledge of the application(s) the user is running. For instance, consider the case of a user running a video streaming application. In order to obtain a sufficient QoS, a minimal end-to-end throughput is needed, as well as a reasonably low jitter. If some of these QoS constraints is violated, the buffer of the video 
streaming application is likely to experience an increasing number of underflows (no frame to be displayed), which results in lower application quality. Hence, violation of QoS constraints on throughput and/or jitter results in buffer underflows, which compromise user-perceived QoS. Similar examples can be done for other classes of user applications.

Note that the degree of user satisfaction is evaluated for both mobile and QoS-driven users. In case the user is mobile, the experienced QoS level is simply tracked and returned as one of the output parameters of the model. In case the user is QoS-driven, the experienced QoS level can influence user mobility (recall Section 3.1).

\section{$4 \quad$ Using WiQoSM}

WiQoSM is written in $\mathrm{C}++$ under a Linux environment. This section describes how WiQoSM can be used to generate user and traffic traces for wireless data networks.

\subsection{Network deployment and user distribution}

Two options are available for AP deployment: guided random and manual. In case of guided random deployment, a number $m$ of APs is distributed in a square deployment region $R$, which is assumed to have a $1 \mathrm{Km}$ long side (this parameter can be arbitrarily set). APs are distributed according to a 'guided uniform' distribution, which is designed to provide at least a certain degree of coverage of the deployment region. More specifically, $R$ is divided into 25 squares of side $200 \mathrm{~m}$, and approximately $\mathrm{m} / 25$ APs are distributed uniformly at random in each square. If $m$ is below 25, $m$ squares are randomly selected in the deployment region, and one AP is distributed uniformly at random in each of the selected squares. Each deployed AP $a$ is then assigned with a degree of popularity $p(a) \in\left[p_{\min }, p_{\max }\right]$, which reflects its potential of attracting network users. The degree of popularity of APs is computed according to a probability distribution resembling a bounded power law (see [13]). Note that other probability distributions for AP popularity can be easily included in our model. In case of manual deployment, both the location and the popularity degree for each AP are provided as input parameters.

After AP deployment, a number $n$ of users is distributed in the network according to the 
following rules. First, each user is assigned a mobility class (QoS-driven/mobile/stationary) and a traffic class (low/medium/high) according to the defined parameters $f, s, l, m$, and $h$. Then, he/she is assigned with an initial position by first randomly selecting an AP according to the popularity metric, and then choosing a position uniformly at random in a bounded vicinity (within the coverage area) of the selected AP. To model users joining/leaving the network, we assume that each user is randomly assigned an active/sleep state, where the active/sleep transition is governed by a Poisson law with parameter $\lambda_{a}$, and the sleep/active transition is governed by a Poisson law with parameter $\lambda_{s}$. Hence, $n$ must be intended as the maximum possible number of network users, and the actual number of users using the network at a given time is in general lower. In order to avoid degenerate situations of excessively long or short transition times, we have imposed an upper and lower bound to the active/sleep and sleep/active transition time. Again, we note that other probability distribution for governing node sleep/active transition can be easily included in our model.

Once all the input parameters has been set and network deployment has been done, WiQoSM can be instructed to generate traffic traces for a certain period of simulated network time.

\subsection{Trace metrics}

An interesting feature of WiQoSM is that the generated traces are accompanied by a set of metrics which can be useful to the network designer to assess some of their relevant features. The metrics computed by WiQoSM are the following:

- total network load (NL): total number of packets exchanged in the network during the simulated time interval.

- data delivery rate: we trace the average data delivery rate experienced by network users during the last $\log$ interval. The data delivery rate is defined as the ratio between the number of bytes correctly sent/received to the number of bytes offered to the network. We also compute the average data delivery rate experienced by network users during the entire simulated time interval. 
- balance index: let $L_{i}$ denote the load observed at the $i$-th AP. The balance index [2] is defined as

$$
\beta=\frac{\left(\sum_{i=1}^{m} L_{i}\right)^{2}}{m \cdot \sum_{i=1}^{m} L_{i}^{2}} .
$$

The balance index is used to measure the deviation in utilization of the different APs: if the load is equally divided amongst the APs, we have $\beta=1$; conversely, with highly unbalanced network load we have $\beta \approx 1 / m$. Similarly to the data delivery rate, the balance index is averaged both in the log interval, and in the entire simulation time. The balance index is computed also for the number of users registered at each AP.

- prevalence and persistence: these metrics, which are used also in [4], model the mobility of users independently of the duration of simulation and of the amount of time a user spends in the network. Prevalence of user $u$, denoted $\operatorname{prev}(u)$, measures the overall fraction of time user $u$ spends with the AP is registered with for the longest period of time. Prevalence accounts for the total registration time with a certain AP, and does not take into account the duration of each session with the given AP. To account for this, we consider also persistence, denoted pers $(u)$, which measures the average amount of time user $u$ stays associated with an access point before being forced to move to another AP or leaving the network. We separately compute these metrics for QoS-driven, mobile, and stationary users.

- distribution of session durations: the simulator also outputs the relative frequency of session durations, discretized to 1 min values. A session is defined as the time elapsing between the instant the user first registers with an AP and the time the user de-registers with the AP. A session can end due to node mobility, change in AP association (only for QoS-driven users), or transition to sleep state.

- Load/User correlation coefficient: for each AP, WiQoSM keeps trace of the number of registered users and of the observed load. These data are used to compute and output the correlation coefficient between number of registered users and load observed at the APs. 
The many parameters used in WiQoSM and their default setting are summarized in Table 2 .

\begin{tabular}{|c|c|c|}
\hline Parameter & Meaning & Default setting \\
\hline \multirow[t]{2}{*}{$m$} & number of APs & 25 \\
\hline & simulated time interval & $14400 \mathrm{sec}$ ( 4 hours) \\
\hline$p(a)$ & degree of popularity of AP $a$ & $\begin{array}{c}\text { chosen according to a power law (guided random) } \\
\text { manually set (manual) }\end{array}$ \\
\hline$n$ & maximum number of users & 400 \\
\hline$\lambda_{a}$ & intensity of Poisson law governing active/sleep transition & 5 min. $(\min )-90 \min .(\operatorname{avg})-240 \min ,(\max )$ \\
\hline$\lambda_{s}$ & intensity of Poisson law governing sleep/active transition & 5 min. (min) $-20 \min .($ avg. $)-90 \min .(\max )$ \\
\hline$r q(u)$ & required QoS value for user $u$ & 0.90 \\
\hline$\Gamma$ & window lenght to determine user satisfaction at current location & 5 sec. \\
\hline$\gamma$ & buffer check interval & $0.05 \mathrm{sec}$ \\
\hline$\lambda_{p}$ & intensity of Poisson law governing pause times at waypoints & 5 min. $(\min )-30 \min .(\operatorname{avg})-120 \min .(\max )$ \\
\hline$v_{\min }, v_{\max }$ & min and max velocity of mobile users & $v_{\min }=v_{\max }=v=1 \mathrm{~m} / \mathrm{sec}$ \\
\hline$f$ & fraction of QoS-driven users & 0.25 \\
\hline$s$ & fraction of stationary users & 0.05 \\
\hline$l, m, h$ & relative fraction of low, medium, and high traffic users & $0.33,0.33,0.33$ \\
\hline pathLoss & radio propagation model & log-distance path loss \\
\hline packSize & packet size (in the data polling model) & $1 K b$ \\
\hline timeSlot & time slot length (in the time polling model) & $200 \mu \mathrm{sec}$ \\
\hline
\end{tabular}

Table 2: Parameters used in the WiQoSM model, and their default setting.

\section{$5 \quad$ WiQoSM assessment}

In this section, we assess WiQoSM ability of producing traces which resemble features observed in traces extracted from real-world WLAN deployments. In particular, we consider sets of traces collected from enterprise and campus environments, whose features have been carefully analyzed in [4] and [27], respectively.

As observed in [17], the process of validating a certain model consists in determining whether the model is an accurate representation of the system. In case of a mobility model generating user and traffic traces such as WiQoSM, model validation consists in comparing certain features of the generated traces with real-world traces. However, to this purpose we cannot use features which are directly accounted for in the model [17], such as the degree of AP popularity in case of WiQoSM: it is clear that such features can easily be reproduced by embedding the properties observed in the real-world trace at hand directly in the model (and, in fact, the way AP popularity is set in WiQoSM is based on the observations reported in [13]). Hence, for WiQoSM assessment we use features of the generated traces that cannot be directly controlled. More 
specifically, we consider some of the statistical properties reported in [4, 27], such as distribution of user prevalence, distribution of session duration, and correlation coefficient between the number of users and load observed at the APs.

We start by describing the main features of the above described statistics reported in $[4,27]$. The traces analyzed in [4] have been collected from a large corporate WLAN spread over three buildings. The WLAN is composed of 177 access points, and traces refers to a four week period of time. During the observed period, 1366 unique users have used the network. The traces analyzed in [27] have been collected in two different periods of time three weeks and 2 months long, respectively. The observed WLAN is located in the ETH campus, and is composed of 166 APs spread over the 32 buildings of the campus. The total number of users observed is 3073 in the first trace, and 4762 in the second trace.

Despite the different WLAN working environment and deployment, the traces analyzed in [4] and in [27] display similar properties for what concerns the distribution of user prevalence and session duration. For what concerns prevalence, it is observed that a relatively large fraction of users (ranging from $25 \%$ to $65 \%$ ) displays very low prevalence (less than $5 \%$ ), implying that the many network users have a relatively high degree of mobility. The relative frequency of users with prevalence higher than $5 \%$ drops considerably, and becomes near zero for values of prevalence higher than 30\%. Finally, a peak in the prevalence distribution, although of moderate amount, can be observed in correspondence of the highest possible value of prevalence (100\%), indicating that a small fraction of the network users (ranging from about 3\% to 10\%) registers always with the same AP when accessing the network. As an example, the prevalence distribution observed in [4] is reproduced in Figure 2, along with the distribution obtained for the trace generated by WiQoSM (details on how WiQoSM trace has been generated are below).

Session duration has been observed to closely resemble a power law in both [4] and [27]. In case of the first ETH trace, short sessions (below 20min) are well approximated by function $0.14 x^{-1.22}$, while relatively longer sessions (30 min or longer) are better approximated by function $x^{-1.4}$. The second ETH trace is well approximated by function $0.14 x^{-1.22}$, independently of session duration. As for the trace analyzed in [4], the best approximating function is $0.92 x^{-1}$ for home users, and by $2.93 x^{-1.36}$ and $34 x^{-1.87}$ for guest users with relatively short (below $100 \mathrm{~min}$ ) and long sessions, respectively. The various approximating functions are summarized in Figure 


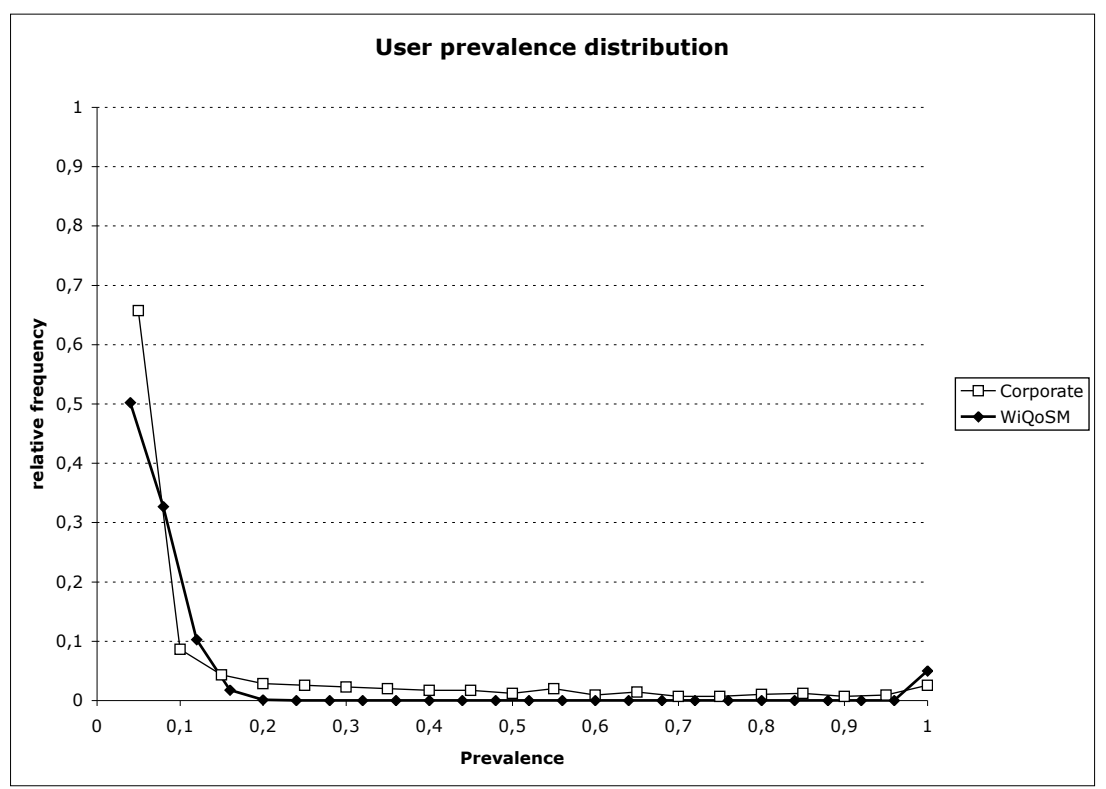

Figure 2: User prevalence distribution reproduced from [4], and for the trace generated by WiQoSM.

3, along with the approximating function obtained for the trace generated by WiQoSM (details on how WiQoSM trace has been generated are below).

In order for WiQoSM to reproduce the above described features of the prevalence and session duration distributions, we observe the following:

a) users with very low prevalence are naturally modeled by mobile users in WiQoSM. Since relatively many users with low prevalence have been observed in $[4,27]$, we need to have relatively many mobile users in WiQoSM. Observe that popularity-based RWP mobility used in WiQoSM tends to play against having many users with low prevalence (because popular APs are expected to be visited more than once by a user), hence we might have to act on the degree of unbalancing in AP popularity in order to faithfully reproduce the target traces.

b) the relatively low (but non-negligible) fraction of users with 100\% prevalence observed in the target traces is well modeled in WiQoSM by stationary users, which have $100 \%$ prevalence by definition. 


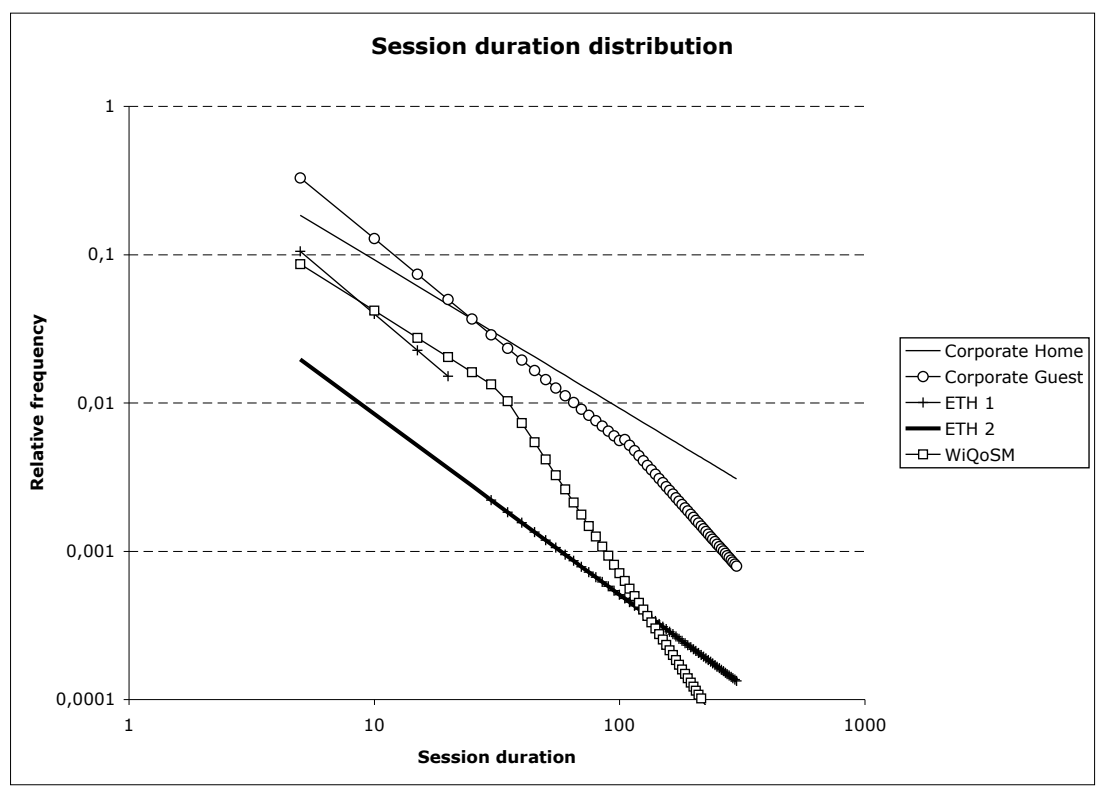

Figure 3: Approximation functions for the session duration distributions observed in [4, 27], and for the trace generated by WiQoSM.

c) in order to have many users with very low prevalence values (5\% or below), we need to simulate a network with a relatively large number of APs: the lower the number of APs, the higher the user prevalence (on the average). This is because, with less possible APs to visit, the relative weight of the most visited one (prevalence) tends to be higher. On the other hand, having more APs contributes negatively to time needed to produce traces. In view of this opposite needs, we have set the number of APs in the following to 100.

d) session duration is determined by three factors: average pause time (mobile users), activation time (all users), and network load (QoS-driven users). In fact, mobile users can end a session due to mobility, or transition into the sleep state; QoS-driven users can end a session due to low QoS-level (which forces either a channel switching or a physical move), or transition into the sleep state; finally, stationary users ends a session only due to transition into the sleep state. Given the above observations, and the fact that the session duration distribution of the target traces call for having a relatively high number of session with short duration (tenths of minutes), and a much lower number of sessions with long duration (up to 5 hours), we need to have a relatively high number of mobile 
users with a relatively short average pause time. Note that this setting of WiQoSM is in accordance to what observed in $a$ ) for what concerns user prevalence distribution. On the other hand, average user activation time must be relatively long, so that the few QoS-driven and stationary users can contribute to the long tail of the session duration distribution.

e) Finally, we observe that the simulated time interval must be relatively long, since we are trying to reproduce statistical features observed in several weeks long traces. Again, we have to deal with the tradeoff between accuracy and simulation running time. Through a set of preliminary simulations, we have observed that generating traces for a six days long period of time provides a reasonable tradeoff.

In view of observations $a$ )-e) above, and after a careful fine tuning, we have set WiQoSM parameters as indicated in Table 3. WiQoSM running time for simulating 1 minute of network operation is $2.5 \mathrm{~s}$ when executed on an Intel Core2Duo E6600 machine with $2 G b$ or RAM ${ }^{4}$. The resulting user prevalence and session duration distributions are shown in figures 2 and 4 , respectively. The best fit functions of the form $c_{1} x^{-c 2}$ for the session duration distribution have been computed with Mathematica ${ }^{\mathrm{TM}}$, and are reported in Figure 3. The values of $c_{1}$ and $c_{2}$ are 0.46 and 1.04 for short sessions (30min and below), and 87.22 and 2.54 for long sessions, respectively. As seen from figures, the trace generated by WiQoSM resembles the statistical features observed in the target traces. For what concerns user prevalence, most of the users have small prevalence values, and a small fraction of users (stationary users) have $100 \%$ prevalence. For what concerns session duration, we observe relatively many sessions with relatively short durations (few tenths of minutes), and relatively few sessions with relatively long durations (up to 5 hours). With respect to the target traces, we observe a somewhat sharper drop of the session duration distribution for relatively long sessions. However, we note that WiQoSM design goal is not mimicking one specific real-world trace (as it is the case for the models introduced in $[13,17,20,21,27])$, but providing a tradeoff between "degree of realism" of the produced traces and allowing flexibility in setting many network parameters.

\footnotetext{
${ }^{4}$ The running time is considerably longer (26s to simulate $60 s$ of network operation) in case of data polling channel access.
} 


\begin{tabular}{|c|c|}
\hline Parameter & Setting \\
\hline$m$ & 100 \\
\hline simulated time & 6 days \\
\hline$p(a)$ & chosen according to a power law in ... \\
\hline$n$ & 1500 \\
\hline$\lambda_{a}$ & $1 \mathrm{~min} .(\min )-240 \min .(\operatorname{avg})-600 \min ,(\max )$ \\
\hline$\lambda_{s}$ & 1 min. (min) $-20 \min .($ avg. $)-90 \min .(\max )$ \\
\hline$r q(u)$ & 0.90 \\
\hline$\Gamma$ & $5 \mathrm{sec}$. \\
\hline$\gamma$ & $0.05 \mathrm{sec}$ \\
\hline$\lambda_{p}$ & $1 \mathrm{~min} .(\min )-20 \min .(\operatorname{avg})-600 \min .(\max )$ \\
\hline$v_{\min }, v_{\max }$ & $v_{\min }=v_{\max }=v=1 \mathrm{~m} / \mathrm{sec}$ \\
\hline QoS mobility rule & unpopular \\
\hline$f$ & 0.20 \\
\hline$s$ & 0.05 \\
\hline$l, m, h$ & $0.33,0.33,0.33$ \\
\hline pathLoss & log-distance path loss $(\alpha=2)$ \\
\hline channel access & time polling \\
\hline timeSlot & $200 \mu \mathrm{sec}$ \\
\hline
\end{tabular}

Table 3: Parameters used to generate the trace whose features are described in figures 3-4.

Figures 2-4 show that WiQoSM is able to produce traces which are "reasonably close" to some of the real-world traces analyzed in the literature. We now demonstrate WiQoSM flexibility: by changing some parameters starting from the configuration reported in Table 3, we show how other relevant parameters of the generated trace (balance indexes and load/user correlation coefficient) varies in a relatively wide range. In particular, Figure 5 shows the effect of varying the fraction of users in the high class of traffic ( $\mathrm{H}$ users) on load and user balance, and on the load/user correlation coefficient. As seen from the figure, while the load balance slightly increases with the percentage of $\mathrm{H}$ users, the user balance tends to decrease with the percentage of $\mathrm{H}$ users. This fact can be explained as follows. The slightly increasing trend of the load balance index indicates that most of the APs are close to saturation. Otherwise, we would have observed a significantly higher value of the load balance index for increasing percentage of $\mathrm{H}$ users (note that $\mathrm{H}$ users are the major cause of traffic asymmetry). Consider now QoS-driven users in the $\mathrm{H}$ traffic class. Due to the high QoS requirement, and given the fact that the network is close to saturation, these users are likely to be unsatisfied. In response to low QoS-level, the unsatisfied QoS-driven user first tries channel switching and, in case channel switching is unsuccessful, he/she physically moves towards a relatively unpopular location. Since the network is close to saturation, we might expect that channel switching is unsuccessful, and unsatisfied QoS-driven H users tend to move to relatively unpopular locations. 


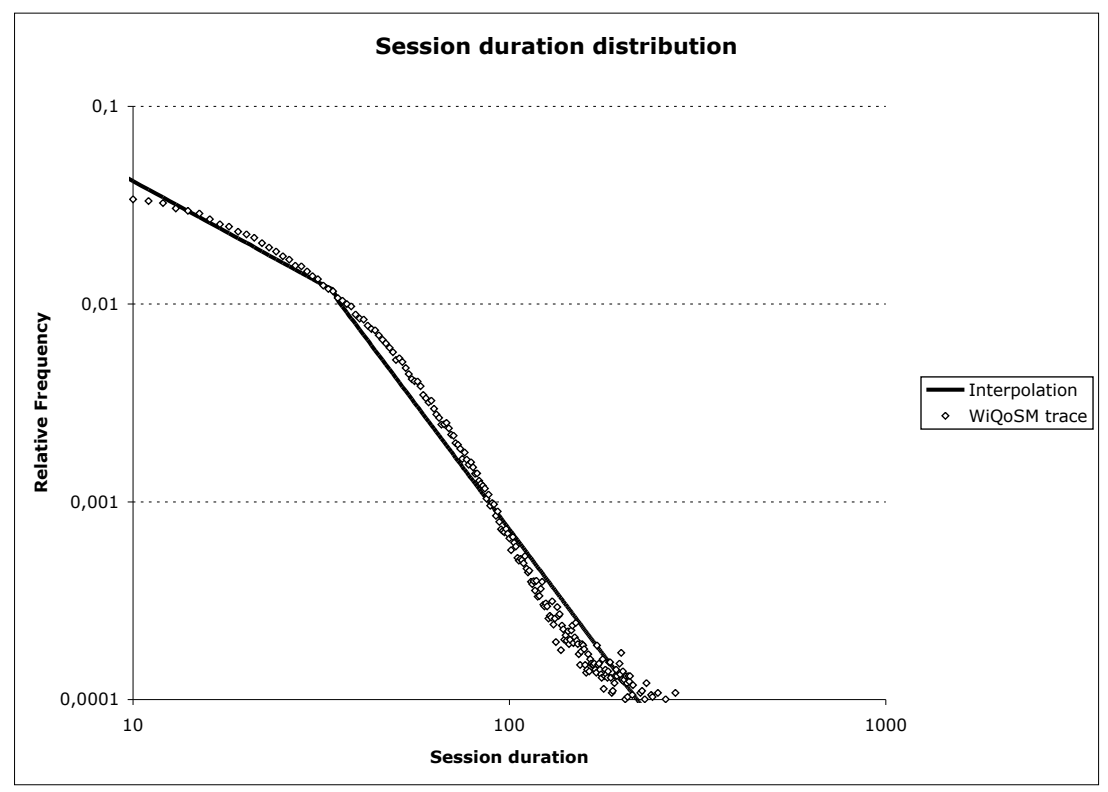

Figure 4: Session duration distribution of the trace generated by WiQoSM.

As a consequence of this, the user balance index is relatively high when the percentage of $\mathrm{H}$ users is low. When the percentage of $\mathrm{H}$ users increases, the number of (unsatisfied) QoSdriven $\mathrm{H}$ users increases as well, and the likelihood of remaining unsatisfied at the unpopular destination point tends to increase (because there are many similar users who tend to move to unpopular locations as well). As a consequence, an unsatisfied QoS-driven $\mathrm{H}$ user is likely to try several channel switching and physical moves before becoming satisfied. The decreasing trend in the user balance index when H increases seems to suggest that an unsatisfied QoSdriven $\mathrm{H}$ user $u$ is likely to become satisfied after a channel switching operation, where the old AP $A$ is likely to be saturated with relatively few users, and the new AP $B$ to which user $u$ registers is likely to be non-saturated with relatively many users. This explains both the decrease in the user balance index when $\mathrm{H}$ increases, and the relatively higher value of the load/user correlation coefficient for relatively high values of $\mathrm{H}$. With respect to correlation, we observe that in the above described channel switching operation we switch from a situation in which correlation is relatively low ( $A$ has relatively few users and relatively high load, and $B$ is in the opposite situation) to a situation in which correlation is increased: after channel switching, $A$ has decreased the number of users but considerably reduced load (because $u$ is a $\mathrm{H}$ 


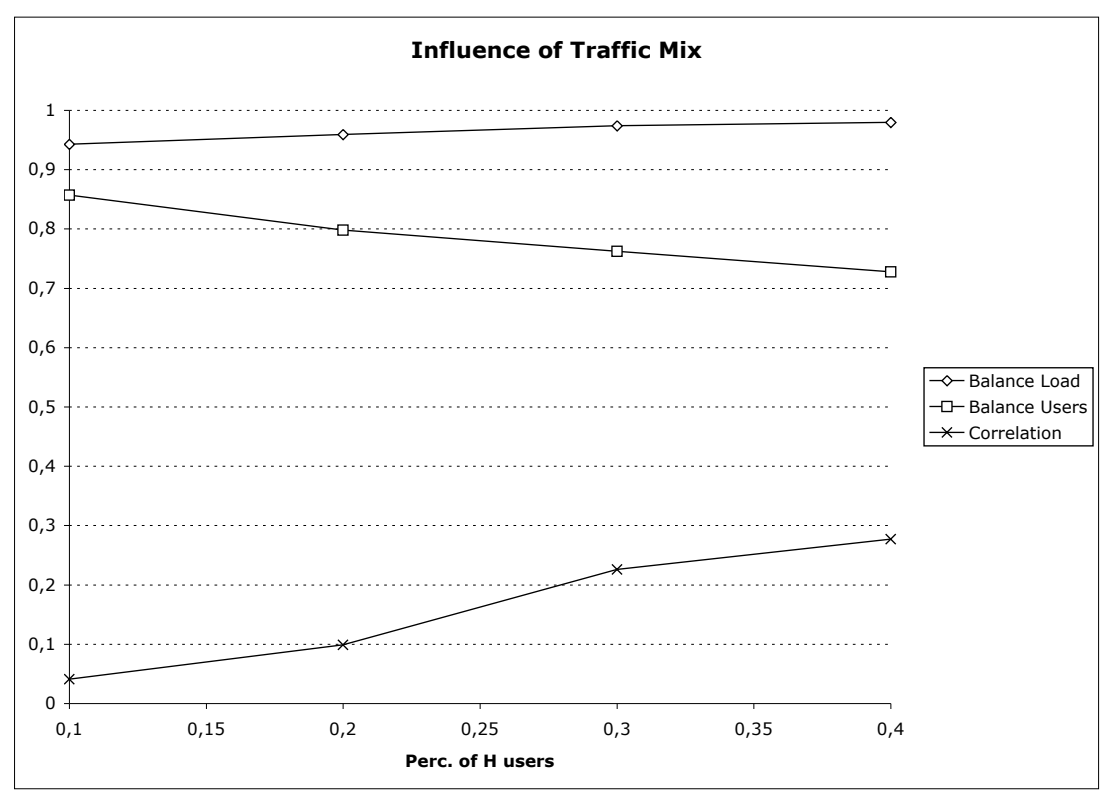

Figure 5: Influence of the traffic mix on load and user balance, and load/user correlation.

user), and $B$ sees a relatively small increase in the number of registered users, but a considerable increase in load.

Two more observations on are in order. First, we have verified that prevalence and session duration distributions are independent of the fraction of users in the $\mathrm{H}$ class. This implies that, at least for what concerns the considered features, WiQoSM allows acting on separate sets of input parameters to careful tuning separate statistics of the output traces. This way, the process of generating synthetic traces which resembles the statistical features of certain target traces is considerably simplified. Second, we observe that the traces generated by WiQoSM closely resembles real-world traces also for what concerns load/user correlation: in fact, the correlation coefficient reported in [4] (the only paper reporting quantitative data for load/user correlation) varies from 0.10 to 0.20 depending on the building considered, and these values are well within the range of correlation values observed in the traces generated by WiQoSM (see Figure 5). 


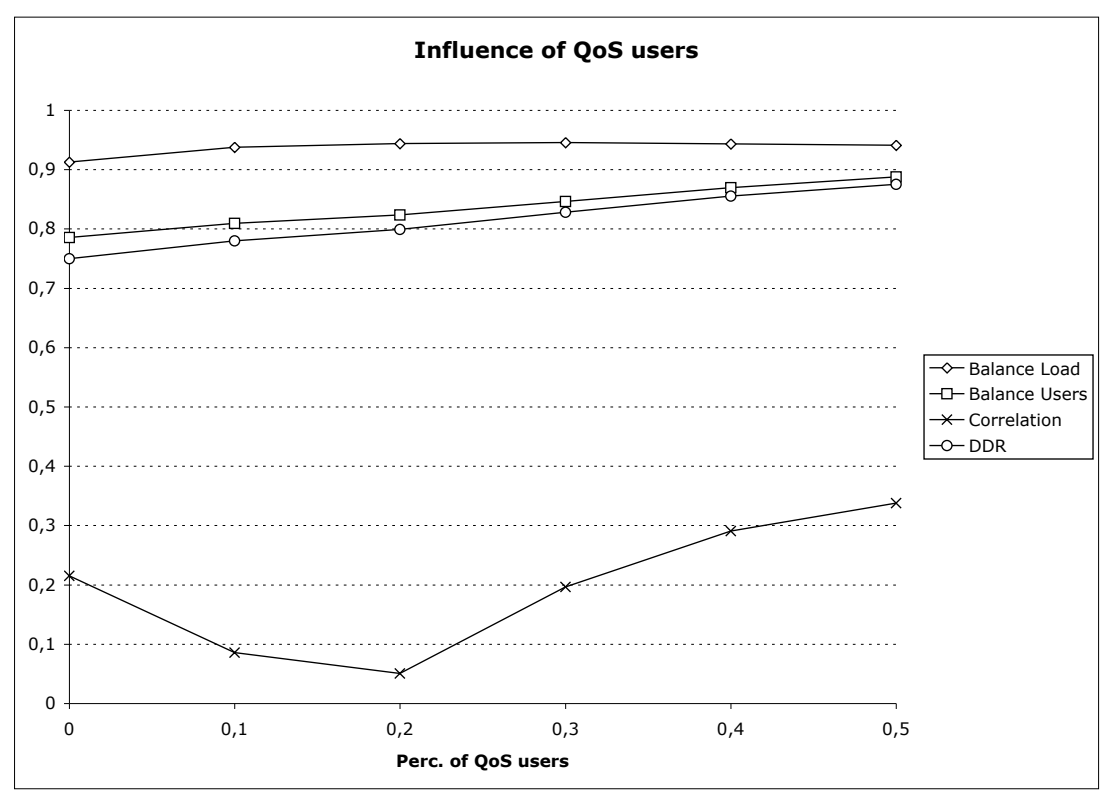

Figure 6: Influence of the fraction of QoS-driven users on load and user balance, and load/user correlation.

\section{$6 \quad$ Influence of QoS-driven users}

One of the novel features of WiQoSM is explicitly modeling QoS-driven users. We have performed a set of simulations to investigate how the fraction of QoS-driven users present in the network affects trace statistics. To reduce simulation running time, we have considered a relatively small network deployment, composed of 25 APs, with a maximum number of users set to 400. The other parameters of the model are set as in Table 2, except for the fraction of QoS-driven users which is varied from 0 to 0.5 . For each setting of the parameters, we have simulated a 1 day time interval, and averaged results over 10 different simulation runs. The results of this set of simulations are reported in Figure 6.

As seen from the figure, both load and user balance index tends to increase with the fraction $f$ of QoS-driven users. This is quite intuitive, since QoS-driven users tend to balance load (because they switch/move from saturated to less saturated APs) and number of registered users (because they tend to move to relatively unpopular locations). The load balance index increases only to a minimal extent, indicating that the network operates close to saturation conditions. 
The behavior of load/user correlation with increasing $f$ is more complex and more difficult to explain. We believe the reason of the initial decreasing trend, followed by an increasing trend, is the following. Unsatisfied QoS-driven users (which are most likely $\mathrm{H}$ users) tend to move to unpopular locations. Hence, these users (who induce a high load on the AP) are likely to be registered with APs with relatively few users. This explains why initially the load/user correlation coefficient decreases with increasing $f$. However, when there are relatively many QoS-driven users, and many of them are unsatisfied, unpopular APs tend to be actually quite popular, because several unsatisfied QoS-driven users tend to move towards these locations. Hence, APs observe a more balanced number of users. Since AP load distribution does not change a lot (network is close to saturation), we have a closer resemblance between user and load distribution, which is reflected by the relatively higher value of the load/user correlation coefficient for relatively high values of $f$. Finally, we observe that, as expected, the presence of QoS-driven users has a positive effect on the average data delivery rate, which is increased from 0.75 to 0.875 when $f$ varies from 0 to 0.5 .

\section{Conclusions}

In this paper, we have introduced the first synthetic mobility and user behavior model which explicitly takes the degree of user satisfaction into account. In particular, in our WiQoSM model a fraction $f$ of the users is assumed to change AP association and/or to move in case the perceived QoS-level drops below an acceptable level.

We have verified that WiQoSM is able to produce traces whose statistical features resemble those observed in some traces collected from real-world WLAN deployments. Furthermore, we have demonstrated WiQoSM ability of allowing fine tuning of disjoint set of parameters, in

order to influence different statistical properties of the generated traces. As such, we believe our model can be successfully used in the simulation of wireless data networks, such as public area WLAN and wireless Mesh deployments. In this respect, our model can be considered as a response to the need of more realistic, yet simple, mobility and user behavior models for wireless hotspots outlined in [3]. With respect to recent models such as those proposed in $[13,17,20,21,27]$, our model is more general and flexible, as several parameters such as number 
of users and APs, traffic mix, fraction of QoS-driven users, and wireless technology at hand can be easily modified. Most importantly, differently from the models of $[13,17,20,21,27]$, WiQoSM produces also load traces for the APs, which can be very useful in testing network performance in presence of variable load.

WiQoSM is intended to be an open source model which can be freely used by the wireless networking community in the simulation of wireless data networks. For this reason, the source code of the model, as well as a very simple user manual, is available on the web at the following URL: http://www.iit.cnr.it/staff/giovanni.resta/WiQoSM. We encourage other researchers to extend and improve WiQoSM in order to incorporate more accurate models of user mobility and/or traffic, other wireless technologies, different QoS models, and so on. In this sense, the model described in this paper must be intended as a starting point, rather than a complete and mature model for wireless data network simulation.

On our side, we are currently working on modeling bursty traffic for users belonging to the low load class. Furthermore, we are planning to use the traces generated by WiQoSM to accurately investigate the performance of different WLAN and wireless Mesh networks protocols.

\section{Acknowledgements}

The authors wish to thank Mauro Leoncini for actively participating in many discussions in the early stage of WiQoSM definition, and Doug Blough for reading a first draft of this paper, and giving several useful suggestions for improving our model. The authors also wish to thank the anonymous reviewers for the many very useful comments and observations, which have considerably improved the quality of this paper.

\section{References}

[1] A. Balachandran, G.M. Voelker, P. Bahl, P. Venkat Rangan, "Characterizing User Behavior and Network Performance in a Public Wireless LAN", Proc. ACM Sigmetrics 02, pp. 195$205,2002$.

[2] A. Balachandran, P. Bahl, G.M. Voelker, "Hot-Spot Congestion Relief and User Service Guarantees in Public-Area Wireless Networks", Proc. IEEE Workshop on Mobile Computing System and Applications (WMCSA), 2002. 
[3] A. Balachandran, G.M. Voelker, P. Bahl, "Wireless Hotspots: Current Challanges and Future Directions", Mobile Networks and Applications, Vol. 10, pp. 265-274, 2005.

[4] M. Balazinska, P. Castro, "Characterizing Mobility and Network Usage in a Corporate Wireless Local-Area Network", Proc. ACM MobiSys 03, pp. 303-316, 2003.

[5] C. Bettstetter, G. Resta, P. Santi, "The Node Distribution of the Random Waypoint Mobility Model for Wireless Ad Hoc Networks", IEEE Trans. on Mobile Computing, Vol. 2, n.3, pp. 257-269, July-Sept. 2003.

[6] C. Bettstetter, "Smooth is Better than Sharp: A Random Mobility Model for Simulation of Wireless Networks", in Proc. ACM Intern. Workshop on Modeling, Analysis, and Simulation of Wireless and Mobile Systems (MSWiM), (Rome, Italy), pp. 19-27, July 2001.

[7] R. Bruno, M. Conti, E. Gregori, "Mesh Networks: Commodity Multihop Ad Hoc Networks", IEEE Communications Magazine, Vol. 43, n. 3, pp. 123-131, March 2005.

[8] T. Camp, J. Boleng, V. Davies, "Mobility models for ad hoc network simulations", Wireless Communication 85 Mobile Computing (WCMC), special issue on mobile ad hoc networking, Wiley, 2002.

[9] Cisco Aironet 1240AG data sheets, available at http://www.cisco.com/en/US/products/hw/ /wireless.

[10] I. Haratcherev, J. Taal, K. Langendoen, R. Legendijk, H. Sips, "Automatic IEEE 802.11 Rate Control for Streaming Applications", Wireless Communications and Mobile Computing, Vol. 5, pp. 421-437, 2005.

[11] M. Heusse, F. Rousseau, G. Berger-Sabbatel, A. Duda, "Performance Anomaly of 802.11b", Proc. IEEE Infocom 03, 2003.

[12] W. Hsu, K. Merchant, H. Shu, C. Hsu, A. Helmy, "Preference-based Mobility Model and the Case for Congestion Relief in WLANs using Ad Hoc Networks", Proc. IEEE VTC 04, 2004.

[13] R. Jain, D. Lelescu, M. Balakrishnan, "Model T: and Empirical Model for User Registration Patterns in a Campus Wireless LAN", Proc. ACM Mobicom, pp. 170-184, 2005.

[14] A. Jardos, E. M. Belding-Royer, K. Almeroth, S. Suri, "Towards Realistic Mobility Models for Mobile Ad Hoc Networks", Proc. ACM MobiCom, pp. 217-229, 2003.

[15] D.B. Johnson, D.A. Maltz, "Dynamic Source Routing in Ad Hoc Wireless Networks", Mobile Computing, Kluwer Academic Publishers, pp. 153-181, 1996.

[16] M. Kim, D. Kotz, "Modeling Users' Mobility among WiFi Access Points", Proc. WiTMeMo 05, 2005.

[17] M. Kim, D. Kotz, S. Kim, "Extracting a Mobility Model from Real User Traces", Proc. IEEE Infocom, 2006. 
[18] D. Kotz, K. Essien, "Characterizing Usage of a Campus-Wide Wireless Network", Proc. ACM Mobicom, pp. 107-118, 2002.

[19] J.Y. LeBoudec, M. Vojnovic, "Perfect Simulation and Stationarity of a Class of Mobility Models", Proc. IEEE Infocom 05, pp. 2743-2754, 2005.

[20] J.K. Lee, J. Hou, "Modeling Steady-State and Transient Behaviors of User Mobility: Formulation, Analysis, and Application", Proc. ACM MobiHoc 06, pp. 85-96, 2006.

[21] D. Lelescu, U. Kozat, R. Jain, M. Balakrishnan, "Model T++: An Empirical Joint SpaceTime Registration Model", Proc. ACM MobiHoc 06, pp. 61-72, 2006.

[22] M. McGuire, "Stationary Distributions of Random Walk Mobility Models for Wireless Ad Hoc Networks", Proc. ACM MobiHoc 05, pp. 90-98, 2005.

[23] M. Musolesi, S. Hailes, C. Mascolo, "An Ad Hoc Mobility Model Founded on Social Network Theory", Proc. ACM MSWiM 04, pp. 20-24, 2004.

[24] W. Navidi, T. Camp, "Stationary Distributions for the Random Waypoint Mobility Model", IEEE Transactions on Mobile Computing, Vol. 3, n. 1, pp. 99-108, 2004.

[25] M. Satyanarayanan, "Pervasive Computing: Vision and Challanges", IEEE Personal Communications, Vol. 8, n. 4, pp. 10-17, August 2001.

[26] D. Tang, M. Baker, "Analysis of a Local-Area Wireless Network", Proc. ACM Mobicom, pp. 1-10, 2000.

[27] C. Tuduce, T. Gross, "A Mobility Model based on WLAN Traces and its Validation", Proc. IEEE Infocom 05, pp. 664-674, 2005.

[28] J. Yoon, M. Liu, and B. Noble, "Random Waypoint Considered Harmful", Proc. IEEE Infocom, (San Francisco, CA), pp. 1312-1321, April 2003.

[29] J. Yoon, M. Liu, and B. Noble, "Sound Mobility Models", Proc. ACM MobiCom, (San Diego, CA), pp. 205-216, Sept. 2003. 